\title{
Evidence from stellar rotation for early disc dispersal owing to close companions ${ }^{\star}$
}

\begin{abstract}
S. Messina
INAF-Catania Astrophysical Observatory, Via S. Sofia, 78, 95123 Catania, Italy

e-mail: sergio.messina@oact.inaf.it

Received 19 November 2018 / Accepted 7 June 2019

ABSTRACT

Context. Young ( $\$ 600 \mathrm{Myr})$ low-mass stars $\left(M \lesssim 1 M_{\odot}\right)$ of equal mass exhibit a distribution of rotation periods. At the very early phases of stellar evolution, this distribution is set by the star-disc locking mechanism, which forces stars to rotate at the same rate as the inner edge of the disc. The primordial disc lifetime and consequently the duration of the disc-locking mechanism, can be significantly shortened by the presence of a close companion, making the rotation period distribution of close binaries different from that of either single stars or wide binaries.

Aims. We use new data to investigate and better constrain the range of ages, the components separation, and the mass ratio dependence at which the rotation period distribution has been significantly affected by the disc dispersal that is enhanced by close companions. Methods. We select a sample of close binaries in the Upper Scorpius association (age 8 Myr) whose components have measured the separation and the rotation periods and compare their period distribution with that of coeval stars that are single stars.

Results. We find that components of close binaries have, on average, rotation periods that are shorter than those of single stars. More precisely, binaries with approximately equal-mass components $\left(0.9 \leq M_{2} / M_{1} \leq 1.0\right)$ have rotation periods that are shorter than those of single stars by $\sim 0.4 \mathrm{~d}$ on average; the primary and secondary components of binaries with smaller mass ratios $\left(0.8<M_{2} / M_{1}<0.9\right)$ have rotation periods that are shorter than those of single stars by $\sim 1.9 \mathrm{~d}$ and $\sim 1.0 \mathrm{~d}$ on average, respectively. A comparison with the older $25 \mathrm{Myr} \beta$ Pictoris association shows that whereas in the latter, all close binaries with projected separation $\rho \leq 80$ AU rotate faster than single stars, in the Upper Scorpius this is only the case for about $70 \%$ of stars.

Conclusions. We interpret the enhanced rotation in close binaries with respect to single stars as the consequence of an early disc dispersal induced by the presence of close companions. The enhanced rotation suggests that disc dispersal timescales are longest for single stars and shorter for close binaries.
\end{abstract}

Key words. stars: low-mass - stars: rotation - binaries: close - open clusters and associations: individual: Upper Scorpius stars: pre-main sequence - stars: variables: T Tauri, Herbig Ae/Be

\section{Introduction}

Late-type stars $\left(M \lesssim 1 M_{\odot}\right)$ with similar mass and age show a distribution of rotation periods. The width of this distribution decreases as stars age, until a one-to-one correspondence between mass and rotation period is reached by an age of about 0.6 Gyr (e.g. Delorme et al. 2011). Such a distribution is thought to arise from a distribution of the initial rotation periods, that is the rotation periods set during the disc-locking phase. Indeed, at the early stages of their life, most if not all stars are characterised by the presence of a primordial circumstellar disc that, while accreting mass and transferring angular momentum onto the star, leaves its imprinting, that is it fixes the value of the initial stellar rotation period. This happens by means of the disclocking mechanism, which forces the outer layers of the star to rotate for a few million years at the rotation rate of the primordial disc inner edge (Shu et al. 1994). After the disc dispersal, this imprinting remains for a long time, until the one-to-one correspondence is reached between mass and period and all memory of the initial rotation period becomes lost.

* Table A.1 is also available at the CDS via anonymous ftp to cdsarc.u-strasbg.fr $(130.79 .128 .5)$ or via http://cdsarc. u-strasbg.fr/viz-bin/qcat?J/A+A/627/A97
Another parameter that effectively contributes to the observed distribution of rotation periods among coeval equalmass stars is the disc lifetime. The primordial disc lifetime is generally not longer than about $10 \mathrm{Myr}$ (Ingleby et al. 2014; Ribas et al. 2014), but there are exceptions (see, e.g. Frasca et al. 2015). However, this lifetime is variable and can be significantly shortened by different factors, such as the gravitational perturbance effects by a close companion. Once a star experiences either an early disc dispersion or inner disc truncation, its rotation rate starts spinning-up earlier than equal-mass disc-bearing stars, because of the radius contraction and angular momentum conservation, gaining a shorter rotation period in comparison.

Indeed, evidence has been accumulated showing that among coeval stars (i.e. members of the same association or cluster) members without discs tend to rotate faster than those with discs (Kraus et al. 2016; Cieza et al. 2009). Furthermore, components of close binaries tend to have a smaller occurrence of discs and to exhibit shorter rotation periods (Stauffer et al. 2016, 2018; Rebull et al. 2018).

We intend to use the rotation period as a diagnostic to explore the effective existence of a disc dispersal enhancement, and its dependence on the separation between the binary components, on their mass ratio, and on age. As already mentioned, the process of enhanced disc dispersal takes place before $10 \mathrm{Myr}$ of age. Therefore, our investigation focuses on clusters and associations 
in this range of ages. Nonetheless, valuable information can also be derived from the analysis of older associations. Indeed, in the $25 \mathrm{Myr}$ beta Pic Association, we found clear evidence for earlier disc dispersal induced by the presence of close companions. At that age, members of close binaries (projected separation $<80 \mathrm{AU}$ ) all rotate faster than their single counterparts (Messina et al. 2017).

Recently, two relevant investigations by Rebull et al. (2018) and Tokovinin \& Briceño (2018) made accurate rotation period measurements available and newly imaged and spatially resolved a number of close binaries in the Upper Scorpius association at an age of $\sim 8 \mathrm{Myr}$. This new information enables us to push our investigation of the effects of binarity on disc dispersal, and then on rotation, back to a much younger age.

In this paper, we report the results of our analysis of the dependence of rotation on binarity at an age of about $8 \mathrm{Myr}$ among the low-mass candidate members of the young stellar association Upper Scorpius (USco). In Sect. 2 we describe the sample selection and the data. In Sect. 3, we present our analysis and in Sect. 4 we present a discussion and our interpretation. Conclusions are given in Sect. 5.

\section{Sample selection}

Rebull et al. (2018) recently measured numerous rotation periods $(\sim 1000)$ in a sample of about 1300 candidate members of the young association USco. Interestingly, they report the finding of a sample of 239 candidate members showing multi-periodic light variations. They inferred periodicities by means of LombScargle periodogram analysis (Scargle 1982) of the photometric time series collected during the Kepler K2 campaign.

Tokovinin \& Briceño (2018) observed by means of spickle interferometry 129 of the brighter stars $(I<13$ mag and $3<$ $\left.(V-K)_{0}<6 \mathrm{mag}\right)$ of the multi-periodic sample of stars found by Rebull et al. (2018). They probed the presence of companions in the separation range from $0.04^{\prime \prime}$ to $\sim 3^{\prime \prime}$, corresponding to separations from $\sim 5$ to $\sim 400 \mathrm{AU}$. As a result of their investigation, they spatially resolved 70 of them, giving additional support to the interpretation that multi-periodic stars are mostly binary stars. The sample selection criterion adopted by Tokovinin \& Briceño (2018) favoured the detection of binaries whose components have comparable flux; indeed most resolved components have a magnitude difference $\Delta I<1 \mathrm{mag}$.

Compared to an isochrone of $8 \mathrm{Myr}$ (Bressan et al. 2012), Tokovinin \& Briceño (2018) found their sample of resolved binaries to be displaced on average by $\simeq+0.75 \mathrm{mag}$ above the isochrone, as expected for binary stars with nearly equal-mass components. They measured $I_{\mathrm{C}}$ magnitude, angular separation, and magnitude difference between the resolved components, and derived the mass of the primary component (see their Table 1).

From the original Tokovinin sample of 70 resolved binaries, we selected a subsample of 49 targets in the colour range $4<$ $(V-K)_{0}<6.5 \mathrm{mag}$ in order to focus on M-type stars.

The close binaries in our sample have mass ratios in the range $0.8 \leq M_{2} / M_{1} \leq 1$, with only one binary with $M_{2} / M_{1}=0.65$. The mass ratio is derived using the mass of the primary component derived by Tokovinin \& Briceño (2018) and the mass $-I_{\text {mag }}$ relation from the $8 \mathrm{Myr}$ isochrone (Bressan et al. 2012), transforming the observed magnitude difference $\Delta I$ into mass difference $\Delta M$

$\Delta M=M_{1}-M_{2}=\Delta I \times 0.197 \pm 0.014$,

where 0.197 is the slope of the mass $-I_{\text {mag }}$ relation in the M0-M6 spectral range.

\section{Analysis}

In the spectral range from M0 to about M6, we investigate if any difference exists between the period distribution of the 49 close binaries with known component separation that we selected from the Tokovinin \& Briceño (2018) sample and the period distribution of all single-star candidate members taken from Rebull et al. (2018).

Rebull et al. (2018) assumed that the periodicity $P 1$ (which corresponds to the highest power peak in the Lomb-Scargle periodogram) is the rotation period of a single star or of the primary component of a multiple system. The second periodicity $P 2$ (which corresponds to the second power peak in the Lomb-Scargle periodogram in order of decreasing power), when detected, is the rotation period of the secondary component of a multiple system. Therefore, $P 1$ and $P 2$ are the rotation periods of the components of the resolved close binaries in our analysis.

The dereddened $(V-K)_{0}$ colours provided by Rebull et al. (2018) for each photometric binary refer to the integrated system, whereas the primary and the secondary components have colours that are bluer and redder, respectively, than the integrated color. To derive the appropriate colours for both components we proceeded as follows. First, we used the Bressan et al. (2012) models for the age of $8 \mathrm{Myr}$ to derive the colour correction $\Delta(V-K)_{0 \mathrm{P}}$ for the primary component,

$\Delta(V-K)_{0 \mathrm{P}}=-2.5 \log \left(\frac{1+\frac{F_{K 2}}{F_{K 1}}}{1+\frac{F_{V 2}}{F_{V 1}}}\right)$,

where $F_{V 1}$ and $F_{V 2}$ are the integrated fluxes in the $V$ band, $F_{K 1}$ and $F_{K 2}$ are those in the $K$ band, and $P$ stands for primary component. We adopted the colours $(V-K)_{0 \mathrm{P}}=(V-K)_{0}+\Delta(V-K)_{0 \mathrm{P}}$ for the primary components.

Similarly, for the secondary component, we computed the colour correction $\Delta(V-K)_{0 \mathrm{~S}}$,

$\Delta(V-K)_{0 \mathrm{~S}}=-2.5 \log \left(\frac{1+\frac{F_{K 1}}{F_{K 2}}}{1+\frac{F_{V 1}}{F_{V 2}}}\right)$,

and adopted the colors $(V-K)_{0 \mathrm{~S}}=(V-K)_{0}+\Delta(V-K)_{0 \mathrm{~S}}$.

The colour correction $\Delta(V-K)_{0 S}$ for the secondary component $(S)$, can also be computed by using the magnitude difference between the components $\Delta I$ measured by Tokovinin \& Briceño (2018) and the linear regression coefficient $(a 1=0.64 \pm 0.07)$ between the observed $I$ magnitude provided by Tokovinin \& Briceño (2018) and the reddening-corrected colour $(V-K)_{0}$, as shown in Fig. 1 :

$\Delta(V-K)_{0 \mathrm{~S}}=0.64 \pm 0.07 \times \Delta I$.

We note that the model values of magnitude and colour from Bressan et al. (2012) yield a larger value for the coefficient $(a 2=0.81 \pm 0.07)$. We find that the use of $a 1$ and $a 2$ produce colours for the secondary components that are 0.08 mag and 0.15 mag redder, respectively, than those computed from Eq. (3). We found that the choice of method used to compute the colours of the secondary components has no effect on the results of the following analysis.

The relevant quantities taken from Tokovinin \& Briceño (2018) and from Rebull et al. (2018) and the new ones computed in the present study for the selected 49 targets are listed in Table A.1.

In Fig. 2 we plot the rotation period $P$ versus $(V-K)_{0}$ colour of all single-period candidate members and overplot the rotation period $P 1$ and $P 2$ of the components of the 49 close 


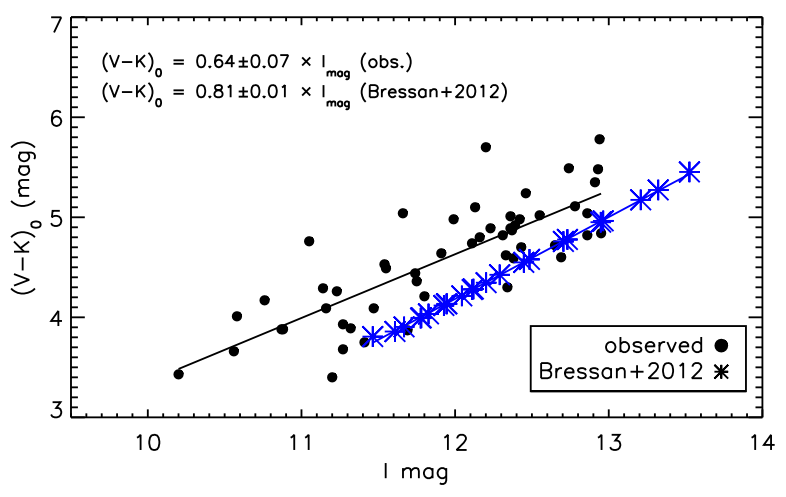

Fig. 1. Colour de-reddened $(V-K)_{0}$ vs. observed $I$ mag (bullets) for the sample observed by Tokovinin \& Briceño (2018). Blue asterisks represent the model $I$ magnitudes corrected for the distance modulus 5.8 mag (Wilkinson et al. 2018), and the model colours from Bressan et al (2012). Solid lines are linear fits. We note that the model values are displaced on average by 0.75 mag from the magnitude of observed binary systems.

binaries versus their colours, as computed according to Eqs. (2) and (3). All stars whose period residuals were larger than $3 \sigma$ (i.e. $P-P_{\text {fit }}>9.2$ d) were rejected (crosses in Fig. 2), and the new fit was computed:

$\log _{10} P=-0.304 \pm 0.020 \times(V-K)_{0}+1.94 \pm 0.10$,

where $P$ is the rotation period in days.

Before proceeding with our analysis, we must consider what follows. In unresolved close binaries, a fainter component whose flux ratio is for example $F_{2} / F_{1} \geq 0.6$ can exhibit activityinduced flux variability with amplitude $\geq 75 \%$ of that exhibited by the primary component. This means that, depending on the specific properties of the activity patterns on the photosphere of both components, the variability arising from the secondary component may be dominant and produce the most powerful peak in the periodogram. In this circumstance the primary period $P 1$ should rather be attributed to the secondary component. Therefore, in our analysis we consider that rotation periods $P 1$ and $P 2$ assigned by Rebull et al. (2018) to the primary and secondary components, respectively, in the case of close binaries with $F_{2} / F_{1} \geq 0.6$, which corresponds to $\Delta I \leq$ $0.5 \mathrm{mag}\left(\Delta M \leq 0.1 M_{\odot}\right)$, may be exchanged.

We compute the difference between the primary periods $P 1$ and the fit (solid line in Fig. 2) and between the secondary period $P 2$ and the fit. This operation allows the mass dependence of the rotation period to be removed. To explore any dependence of the rotation enhancement on the mass ratio between the components of a binary system, we consider two different mass ratio ranges: binaries whose components have about equal mass $M_{2} / M_{1} \geq 0.9$ and binaries with smaller mass ratios $0.8 \leq M_{2} / M_{1}<0.9$. The rotation period residuals $P_{\mathrm{obs}}-P_{\text {fit }}$ are plotted versus the projected separation of the components in Fig. 3. As previously done for the single stars, components of binaries with period residuals larger than $3 \sigma$ are also excluded from the following analysis (crossed symbols in Fig. 2). The fact that the component of one binary has an outlying period does not necessarily imply that the binary is not a member of the USco association. For example, the spot pattern on that component may have lead to measurement of the beat period instead of the rotation period. Therefore, we only excluded the outlying component from the analysis while keeping the rotation period of the other component.

In the case of close binaries with components of about equalmass, as explained above, we do not know to which components the $P 1$ and $P 2$ rotation periods refer. Therefore, we decided to make Monte Carlo simulations where the $P 1$ and $P 2$ periods of each binary are randomly permuted. We made 1000 such simulations and for each we measured the average $\left\langle P 1-P_{\text {fit }}\right\rangle$ and $\left\langle P 2-P_{\text {fit }}\right\rangle$. The results of our simulations are plotted in the inner plots of the top panel of Fig. 3, where we plot in the form of histograms the distribution of the average $\left\langle P-P_{\text {fit }}\right\rangle$ for each of the 1000 simulations. We find that both periods $P 1$ and $P 2$ are shorter by $\simeq 0.4 \mathrm{~d}$ with respect to the average periods of single stars. We also find that the residuals $P 1$-fit and $P 2$-fit show some marginal evidence to be correlated to the projected separation between the binary components (top panel of Fig. 3). The Spearman's rank correlation analysis gives similar correlation coefficient $\rho=+0.29$ and significance $p$-value $=0.14$ for the $P 1-$ fit and the $P 2-$ fit.

In the case of non-equal-mass components (bottom panel of Fig. 3), we find that both periods $P 1$ and $P 2$ are shorter by $\simeq 1.9 \mathrm{~d}$ and $\simeq 1.0 \mathrm{~d}$, respectively, compared to the average period of single stars. The Spearman's rank correlation analysis gives a correlation coefficient $\rho=+0.17$ and a significance $p$-value $=0.43$ for $P 1-$ fit and $\rho=-0.11$ and a significance $p$-value $=0.63$ for $P 2-$ fit between residuals and projected separation.

To summarise, we find that candidate members of USco in close binaries (median separation $\sim 21$ AU in the analysed sample) rotate faster than their single counterparts; moreover, the lower-mass components of non-equal-mass binaries tend to have the shortest rotation periods. We find some marginal evidence that the closer the equal-mass binary components, the faster their rotation period with respect to single stars.

Another property of our sample of close binaries, in addition to the average rotation period, is the period difference between the two components. We have seen that the rotation period is mass dependent; therefore, the period difference between the two components of the same system may arise on only their mass difference. For this reason, we first remove the mass dependence by computing the residuals with respect to a linear fit to the periods before computing the period differences. We find that the average period difference between components of approximately equal-mass binaries $\left(M_{2} / M_{1} \geq 0.9\right)$ is $\langle\Delta P\rangle \simeq 0.8 \mathrm{~d}$ (with a dispersion $\sigma=1.6 \mathrm{~d}$ ) against $\langle\Delta P\rangle \simeq 0.2 \mathrm{~d}$ (with a dispersion $\sigma=1.9 \mathrm{~d}$ ) between the components of unequal-mass binaries $\left(0.8 \leq M_{2} / M_{1}<0.9\right)$.

The last property that we take into consideration is the width of the period distribution (see Fig. 4). After removing the mass dependence, as already done before, we find that among binaries with approximately equal-mass components, primary and secondary components have residual distribution, respectively, smaller $(\sigma \simeq 0.7 \mathrm{~d})$ and larger $(\sigma \simeq 1.1 \mathrm{~d})$ than single stars $(\sigma \simeq 0.9 \mathrm{~d})$. Alternatively, among binaries with non-equal-mass components, secondaries have similar residual distribution to single stars $(\sigma \simeq 0.9 \mathrm{~d})$, whereas primaries have a larger width of the residual distribution with a standard deviation $\sigma \simeq 1.1 \mathrm{~d}$.

Another important aspect concerns the fraction of discs. In the colour range under analysis, about $28 \%$ of candidate members that are single (i.e. with only one periodicity measured) show strong evidence for a disc. If we consider the resolved close binaries in the same colour range, we find that the fraction decreases to $14 \%$.

\section{Discussion}

The disc lifetime is variable, but generally not longer than $\sim 10$ Myr (Ingleby et al. 2014; Ribas et al. 2014). Theories supported by observations predict that the disc lifetime can 


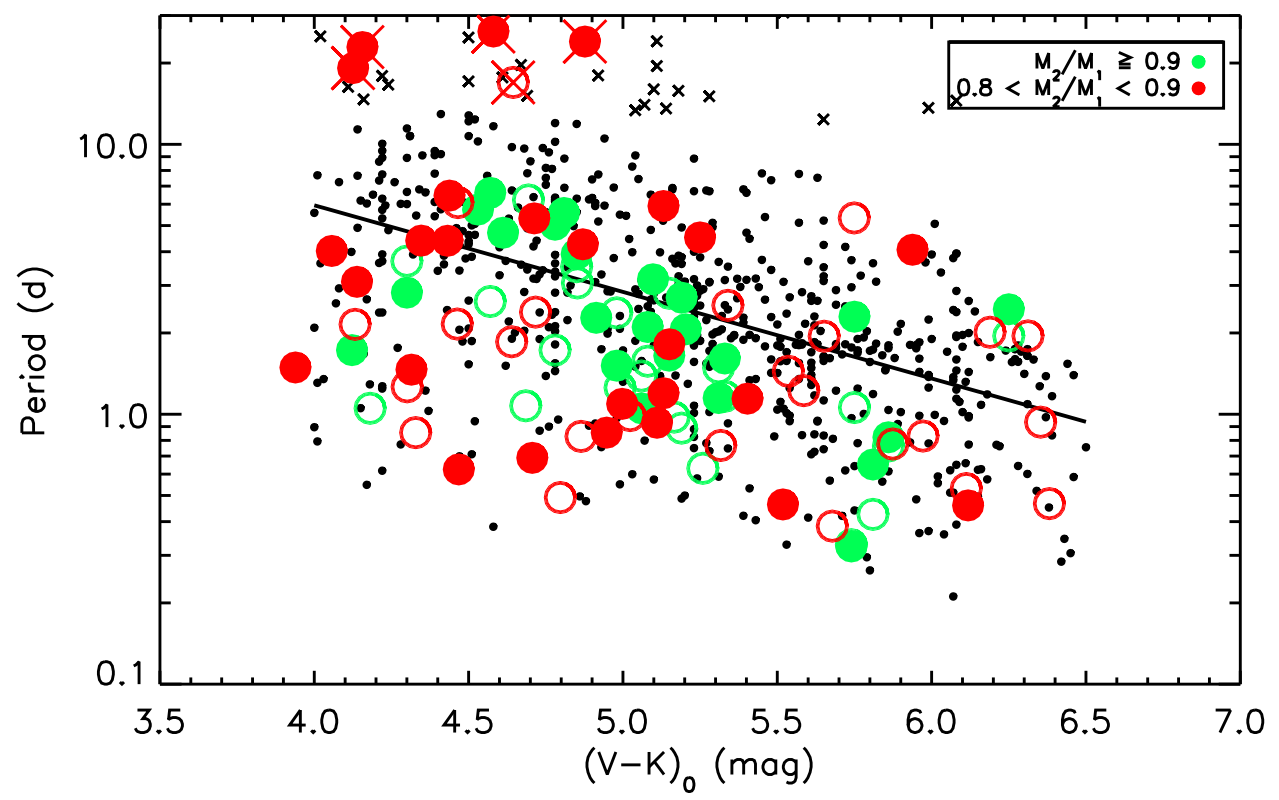

Fig. 2. Distribution of stellar rotation periods vs. dereddened colour for candidate members of the Upper Scorpius association from Rebull et al. (2018) in the M0-M6 spectral range. Small dots are the rotation periods of single stars as listed in Table 1 of Rebull et al. (2018). Filled and open bullets are periods $P 1$ and $P 2$ of components of close binaries, respectively. Components of binary systems whose mass ratio is $M_{2} / M_{1} \geq 0.9$ (respectively $0.8 \leq M_{2} / M_{1} \leq 0.9$ ) are shown in green (respectively red). The solid line is a fit to the rotation periods of single stars (see Eq. (5)). Crossed symbols are outliers excluded from the fit computation and the following analysis.
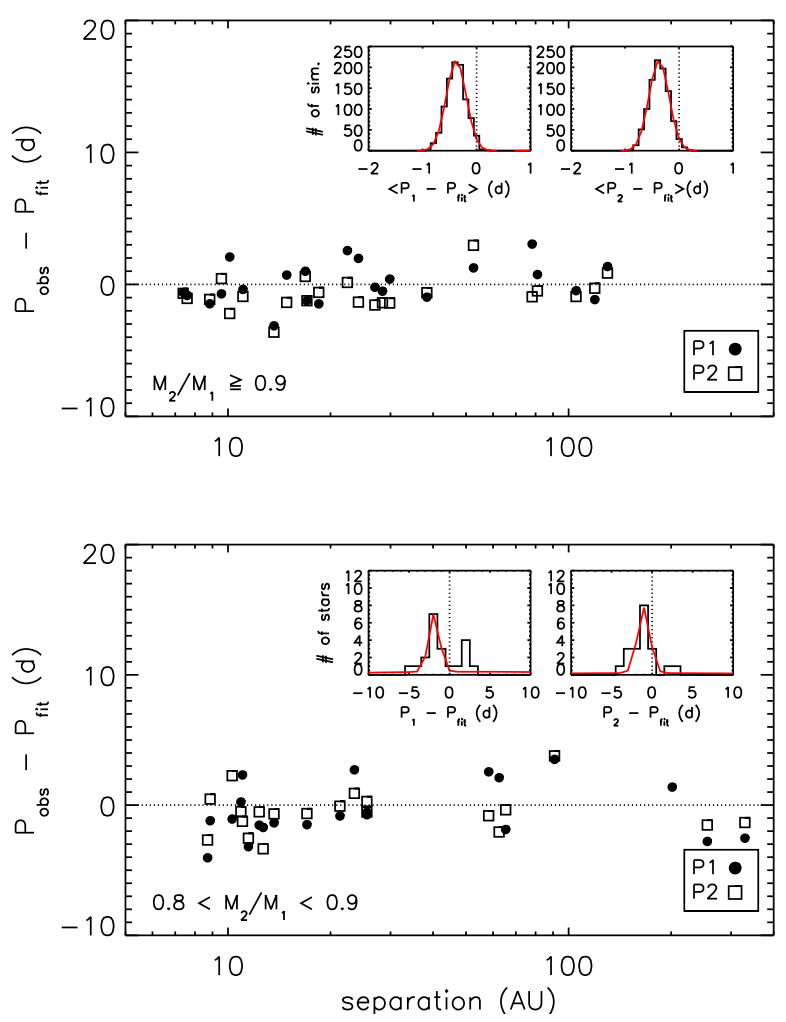

Fig. 3. Distribution of residuals of periods with respect to the fit (solid line in Fig. 2) for close binaries with about equal-mass components $M_{2} / M_{1} \geq 0.9$ (top panel) and with non-equal-mass components $0.8 \leq$ $M_{2} / M_{1}<0.9$ (bottom panel). The top inner plots show the distribution of average $\left\langle P-P_{\text {fit }}\right\rangle$ residuals from Monte Carlo simulations (see text for explanation) with a Gaussian fit over plotted; the bottom inner plots show the distribution of the $P-P_{\text {fit }}$ residuals.

be significantly shortened by the presence of a companion (Meibom et al. 2007; Bouvier et al. 1993; Edwards et al. 1993; Ingleby et al. 2014; Rebull et al. 2004). Kraus et al. (2016) and Cieza et al. (2009) found that stars without IR excess tend to have companions at smaller separation than stars with excess indicating the presence of a disc. Both studies find that the depletion of primordial discs among binary systems with components

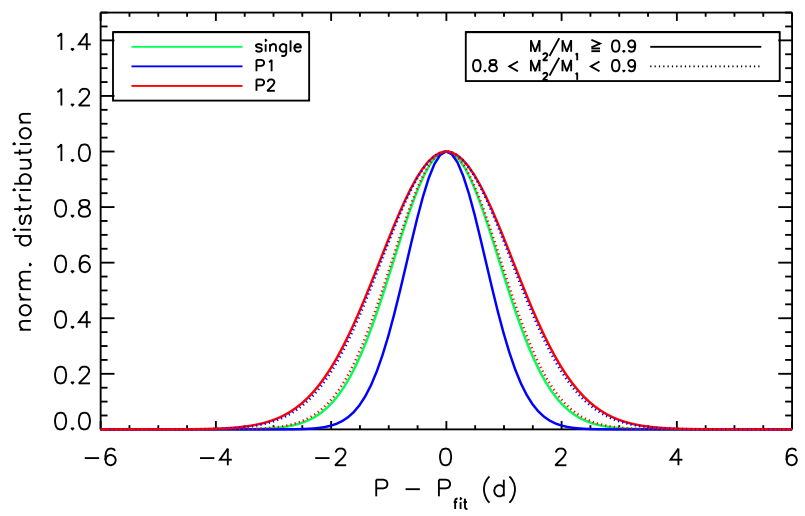

Fig. 4. Gaussian fits to the distribution of the residuals of the rotation periods: green line for single stars, blue line for primary components, and red line for secondary components of binary systems. We note that the distributions for the $P 1$ and $P 2$ of binary components have been shifted to be centered on zero to make the width difference more easily readable.

closer than $40 \mathrm{AU}$ is a factor of two larger than in either single or wide binaries already at ages as young as 1-2 Myr. Stauffer et al. (2016) report that photometric binaries among the Pleiades GKM-type stars tend to rotate faster than their counterpart single stars. Douglas et al. (2016) report that most, if not all, rapid rotators that deviate from the single-valued relation between mass and rotation already reached by the age of the Hyades ( $\sim 6$ Gyr), belong to multiple systems.

Recently, Messina et al. (2017), analysing the rotation period distribution of the members of the 25 Myr beta Pic Association, found that single stars and components of multiple systems with projected separation larger than about $80 \mathrm{AU}$ have similar distribution of rotation periods versus $V-K_{s}$ colour. On the contrary, components of close visual binaries/triplets with projected separation smaller than about $80 \mathrm{AU}$ rotate preferentially faster than their equal-mass single counterparts. This circumstance suggests that when the components are sufficiently close, their primordial discs undergo an enhanced dispersal allowing the stars to start their spin-up earlier than single stars.

The results by Messina et al. (2017) for stars of $25 \mathrm{Myr}$ of age can be compared to those we found for stars of $8 \mathrm{Myr}$ 


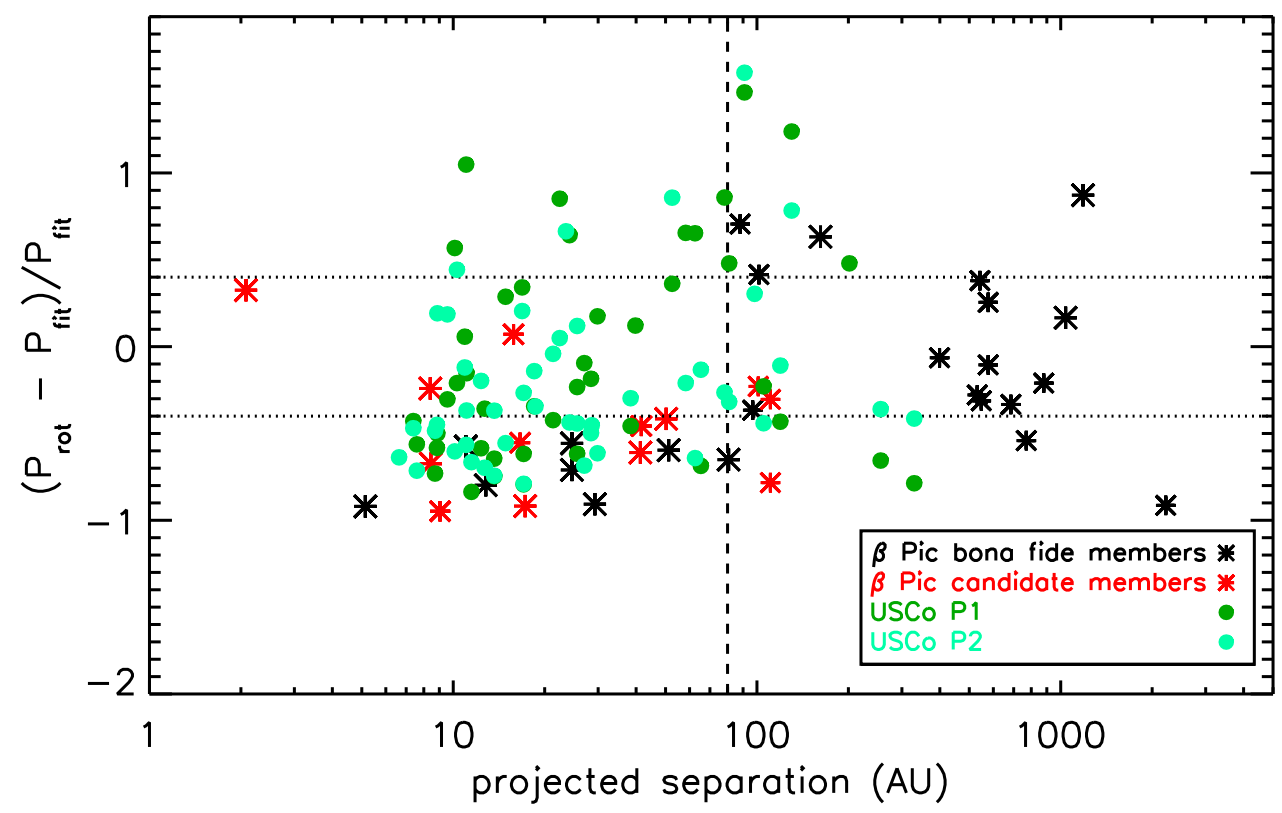

Fig. 5. Comparison between the distribution of relative period residuals vs. projected separation for the members of the $\beta$ Pic association (see also Fig. 7 of Messina et al. 2017) and the close-binary candidate members of Upper Scorpius. Horizontal dotted lines represent the width $( \pm 3 \sigma)$ of the period distribution of the $\beta$ Pic single members. In the $\beta$ Pic association, close binaries with component separation $<80 \mathrm{AU}$ (vertical dashed line) mostly rotate faster than single stars. for the USco association. In Fig. 5, we plot the results of Messina et al. (2017), that is the relative residuals of the fit to the rotation period versus the projected separation (AU) as asterisks and overplot the same quantity but for the resolved binaries considered in this study as bullets. The use of residuals allows us to remove the mass dependence in the period distribution. We note that in the 5-80 AU range of projected separation about $70 \%$ of close binaries at the younger age of USco have periods shorter (negative residuals) than their single counterparts, and about $30 \%$ still have periods comparable to those of the single stars. Conversely, at the older age of the $\beta$ Pic association, all close binaries have periods shorter than their single counterparts. This is a clear indication that the post-disc dispersal stellar rotation spin up is already set at ages younger than $8 \mathrm{Myr}$, and that it has produced measurable effects on the majority of close binaries by an age of $8 \mathrm{Myr}$. The disc dispersal timescale in these close binaries must be different from binary to binary, with a range of values, allowing for close binaries (about 30\% in this sample) where the dispersal takes place slowly, making the rotation spinup not yet effective, as well as binaries where the dispersal was quite sudden making the rotation spin-up measurable.

We also note that the rotation-period shortening for components of equal-mass binaries $(\sim 0.4 \mathrm{~d}$ for $P 1$ and $P 2)$ is smaller than for non-equal-mass binaries $(\sim 1.9 \mathrm{~d}$ for $P 1$ and $\sim 1.0 \mathrm{~d}$ for $P 2)$. This suggests that the timescale of their disc dispersal is dependent on the mass ratio between the binary components.

\section{Conclusions}

We analysed a sample of 49 close binaries that are candidate members of the Upper Scorpius association whose components are in the M0-M6 spectral range, and have known rotation periods and projected separations $(\rho<100 \mathrm{AU})$. We found clear evidence that they rotate faster than their single counterparts. On average, components of close binaries exhibit rotation periods shorter by an amount ranging from $0.4 \mathrm{~d}$ if they have about equal-mass, to $1.9 \mathrm{~d}$ as in the case of the lower-mass components of lower mass-ratio binaries. The rotation spin up of close binaries with respect to single stars can be attributed, among different processes, to an early dispersion or truncation of the primordial circumstellar disc owing to gravitational effects by the close companion. Such a disc dispersal likely starts operating in the very first few million years of stellar life, producing measurable effects at the age of $8 \mathrm{Myr}$.

In our hypothesis that the rotation period shortening with respect to single stars is a direct consequence of early disc dispersal, we infer that the timescale of disc dispersal is the longest in single stars or in wide-orbit $(\rho \gtrsim 100 \mathrm{AU})$ components of multiple systems, is shorter in binaries with about equal-mass components, and is even shorter in binaries with non-equal-mass components. Finally, we find that components of about equal-mass and of nonequal-mass binaries generally have different rotation period dispersion widths, where primary components of equal-mass binaries exhibit a smaller dispersion than that of single stars, whereas secondaries and components of non-equal-mass binaries all exhibit dispersion comparable to or larger than that of single stars.

Acknowledgements. Research on stellar activity at INAF- Catania Astrophysical Observatory is supported by MIUR (Ministero dell'Istruzione, dell'Università e della Ricerca). This research has made use of the Simbad database operated at CDS (Strasbourg, France). We are grateful to the referee who allowed us to significantly improve the quality of this paper.

\section{References}

Bouvier, J., Cabrit, S., Fernandez, M., Martin, E. L., \& Matthews, J. M. 1993, A\&A, 272, 176

Bressan, A., Marigo, P., Girardi, L., et al. 2012, MNRAS, 427, 127

Cieza, L. A., Padgett, D. L., Allen, L. E., et al. 2009, ApJ, 696, L84 Delorme, P., Collier Cameron, A., Hebb, L., et al. 2011, MNRAS, 413, 2218

Douglas, S. T., Agüeros, M. A., Covey, K. R., et al. 2016, ApJ, 822, 47

Edwards, S., Strom, S. E., Hartigan, P., et al. 1993, AJ, 106, 372

Frasca, A., Biazzo, K., Lanzafame, A. C., et al. 2015, A\&A, 575, A4

Ingleby, L., Calvet, N., Hernández, J., et al. 2014, ApJ, 790, 47

Kraus, A. L., Ireland, M. J., Huber, D., Mann, A. W., \& Dupuy, T. J. 2016, AJ, 152,8

Meibom, S., Mathieu, R. D., \& Stassun, K. G. 2007, ApJ, 665, L155

Messina, S., Lanzafame, A. C., Malo, L., et al. 2017, A\&A, 607, A3

Rebull, L. M., Wolff, S. C., \& Strom, S. E. 2004, AJ, 127, 1029

Rebull, L. M., Stauffer, J. R., Cody, A. M., et al. 2018, AJ, 155, 196

Ribas, Á., Merín, B., Bouy, H., \& Maud, L. T. 2014, A\&A, 561, A54

Scargle, J. D. 1982, ApJ, 263, 835

Shu, F., Najita, J., Ostriker, E., et al. 1994, ApJ, 429, 781

Stauffer, J., Rebull, L., Bouvier, J., et al. 2016, AJ, 152, 115

Stauffer, J., Rebull, L., Cody, A. M., et al. 2018, AJ, 156, 275

Tokovinin, A., \& Briceño, C. 2018, AJ, 156, 138

Wilkinson, S., Merín, B., \& Riviere-Marichalar, P. 2018, A\&A, 618, A12 


\section{Appendix A: Additional table}

Table A.1. EPIC ID number of the 49 binaries considered in the present study.

\begin{tabular}{|c|c|c|c|c|c|c|c|c|c|c|}
\hline $\begin{array}{c}\text { EPIC } \\
\text { ID }\end{array}$ & $\begin{array}{c}(V-K)_{0}{ }^{(a)} \\
(\mathrm{mag})\end{array}$ & $\begin{array}{c}(V-K)_{\mathrm{OP}} \\
(\mathrm{mag})\end{array}$ & $\begin{array}{c}(V-K)_{0 \mathrm{~S}} \\
(\mathrm{mag})\end{array}$ & $\begin{array}{c}M_{1}{ }^{(b)} \\
\left(M_{\odot}\right)\end{array}$ & $\begin{array}{c}M_{2} \\
\left(M_{\odot}\right)\end{array}$ & $\begin{array}{c}P 1^{(a)} \\
(\mathrm{d})\end{array}$ & $\begin{array}{c}P 2^{(a)} \\
\text { (d) }\end{array}$ & $\begin{array}{c}I \operatorname{mag}^{(b)} \\
(\mathrm{mag})\end{array}$ & $\begin{array}{l}\Delta I^{(b)} \\
(\mathrm{mag})\end{array}$ & $\begin{array}{r}\text { Separation }^{(b)} \\
(\mathrm{AU})\end{array}$ \\
\hline EPIC 203553934 & 4.65 & 4.61 & 4.69 & 0.59 & 0.55 & 4.70 & 6.22 & 11.47 & 0.20 & 52.57 \\
\hline EPIC 204918279 & 6.23 & 6.12 & 6.38 & 0.29 & 0.25 & 0.46 & 0.47 & 12.60 & 0.20 & 25.57 \\
\hline EPIC 204104740 & 4.83 & 4.81 & 4.85 & 0.56 & 0.54 & 5.57 & 3.06 & 11.55 & 0.10 & 22.41 \\
\hline EPIC 204832936 & 4.98 & 4.87 & 6.20 & 0.38 & 0.03 & 4.26 & 4.98 & 12.86 & 1.80 & 201.46 \\
\hline EPIC 204477741 & 5.86 & 5.86 & 5.86 & 0.31 & 0.31 & 0.82 & 0.76 & 12.74 & 0.00 & 7.39 \\
\hline EPIC 204878974 & 4.22 & 4.14 & 4.33 & 0.56 & 0.48 & 3.09 & 0.85 & 11.16 & 0.40 & 12.68 \\
\hline EPIC 204350593 & 5.13 & 5.10 & 5.17 & 0.42 & 0.40 & 3.16 & 0.98 & 12.16 & 0.10 & 14.87 \\
\hline EPIC 204406748 & 4.52 & 4.43 & 4.64 & 0.69 & 0.57 & 4.39 & 16.96 & 11.14 & 0.60 & 39.78 \\
\hline EPIC 203690414 & 5.33 & 5.15 & 5.65 & 0.32 & 0.24 & 1.82 & 1.95 & 12.95 & 0.40 & 25.57 \\
\hline EPIC 204794876 & 4.02 & 3.94 & 4.13 & 0.73 & 0.61 & 1.49 & 2.15 & 10.87 & 0.60 & 8.71 \\
\hline EPIC 204862109 & 4.15 & 4.12 & 4.18 & 0.73 & 0.69 & 1.73 & 1.05 & 10.88 & 0.20 & 13.64 \\
\hline EPIC 204637622 & 5.06 & 5.06 & 5.06 & 0.50 & 0.50 & 1.05 & 1.39 & 11.99 & 0.00 & 8.84 \\
\hline EPIC 204603210 & 5.19 & 5.00 & 5.59 & 0.39 & 0.27 & 1.09 & 1.22 & 12.42 & 0.60 & 12.35 \\
\hline EPIC 204856827 & 5.15 & 5.15 & 5.15 & 0.44 & 0.44 & 1.65 & 2.81 & 12.13 & 0.00 & 9.56 \\
\hline EPIC 204242152 & 5.08 & 5.08 & 5.08 & 0.45 & 0.45 & 2.10 & 1.57 & 12.33 & 0.00 & 11.06 \\
\hline EPIC 204757338 & 5.75 & 5.75 & 5.75 & 0.30 & 0.30 & 2.30 & 1.06 & 12.94 & 0.00 & 80.98 \\
\hline EPIC 204845955 & 5.22 & 5.13 & 5.34 & 0.53 & 0.45 & 1.20 & 2.53 & 11.66 & 0.40 & 8.86 \\
\hline EPIC 204229583 & 4.89 & 4.71 & 5.32 & 0.55 & 0.35 & 0.69 & 0.77 & 11.54 & 1.00 & 329.27 \\
\hline EPIC 203851147 & 5.33 & 5.13 & 5.75 & 0.38 & 0.26 & 5.91 & 5.34 & 12.37 & 0.60 & 90.92 \\
\hline EPIC 205087483 & 4.98 & 4.98 & 4.98 & 0.46 & 0.46 & 1.51 & 2.38 & 12.23 & 0.00 & 119.34 \\
\hline EPIC 205177770 & 5.65 & 5.40 & 6.35 & 0.30 & 0.18 & 1.14 & 0.93 & 12.91 & 0.60 & 21.31 \\
\hline EPIC 204429883 & 5.74 & 5.74 & 5.74 & 0.38 & 0.38 & 0.32 & 0.33 & 12.20 & 0.00 & 17.05 \\
\hline EPIC 204608292 & 4.50 & 4.35 & 5.97 & 0.36 & 0.10 & 4.40 & 0.83 & 12.38 & 1.30 & 10.91 \\
\hline EPIC 203036995 & 5.33 & 5.11 & 5.87 & 0.38 & 0.24 & 0.93 & 0.78 & 12.39 & 0.70 & 17.05 \\
\hline EPIC 203716047 & 5.14 & 4.95 & 5.54 & 0.39 & 0.27 & 0.85 & 1.44 & 12.43 & 0.60 & 65.35 \\
\hline EPIC 204857023 & 4.44 & 4.31 & 4.64 & 0.54 & 0.42 & 1.46 & 1.86 & 12.46 & 0.60 & 255.36 \\
\hline EPIC 203048597 & 5.33 & 5.33 & 5.33 & 0.39 & 0.39 & 1.61 & 1.17 & 12.31 & 0.00 & 105.13 \\
\hline EPIC 204156820 & 4.57 & 4.57 & 4.57 & 0.59 & 0.59 & 6.61 & 2.62 & 11.74 & 0.00 & 78.14 \\
\hline EPIC 205225696 & 5.19 & 5.19 & 5.19 & 0.34 & 0.34 & 2.70 & 0.89 & 13.26 & 0.00 & 29.83 \\
\hline EPIC 203777800 & 4.95 & 4.91 & 4.99 & 0.39 & 0.37 & 2.28 & 1.25 & 12.65 & 0.10 & 28.41 \\
\hline EPIC 204449800 & 6.25 & 6.25 & 6.25 & 0.31 & 0.31 & 2.45 & 1.95 & 12.58 & 0.00 & 130.05 \\
\hline EPIC 204235325 & 5.11 & 4.88 & 6.19 & 0.44 & 0.20 & 24.03 & 2.01 & 12.11 & 1.20 & 98.19 \\
\hline EPIC 202533810 & 4.78 & 4.78 & 4.78 & 0.52 & 0.52 & 5.04 & 1.73 & 11.80 & 0.00 & 24.15 \\
\hline EPIC 204204606 & 5.31 & 5.31 & 5.31 & 0.33 & 0.33 & 1.15 & 1.49 & 12.86 & 0.00 & 38.36 \\
\hline EPIC 204082531 & 5.81 & 5.81 & 5.81 & 0.27 & 0.27 & 0.65 & 0.43 & 12.78 & 0.00 & 7.59 \\
\hline EPIC 203855509 & 5.41 & 5.25 & 5.68 & 0.35 & 0.27 & 4.52 & 0.39 & 12.93 & 0.40 & 11.03 \\
\hline EPIC 203809317 & 4.55 & 4.44 & 4.72 & 0.52 & 0.42 & 6.46 & 2.39 & 12.36 & 0.50 & 58.25 \\
\hline EPIC 204569229 & 4.22 & 4.16 & 4.30 & 0.66 & 0.58 & 22.92 & 1.26 & 11.27 & 0.40 & 6.63 \\
\hline EPIC 204655550 & 4.20 & 4.06 & 4.47 & 0.63 & 0.45 & 4.03 & 6.08 & 11.41 & 0.90 & 10.28 \\
\hline EPIC 204374147 & 4.59 & 4.47 & 4.80 & 0.71 & 0.53 & 0.62 & 0.49 & 11.05 & 0.90 & 11.48 \\
\hline EPIC 203001867 & 4.78 & 4.71 & 4.86 & 0.53 & 0.47 & 5.32 & 0.83 & 11.91 & 0.30 & 62.51 \\
\hline EPIC 204462113 & 4.60 & 4.53 & 4.69 & 0.40 & 0.36 & 5.73 & 1.07 & 12.69 & 0.20 & 10.12 \\
\hline EPIC 203071614 & 5.72 & 5.52 & 6.11 & 0.29 & 0.21 & 0.46 & 0.53 & 12.55 & 0.40 & 13.65 \\
\hline EPIC 204520585 & 4.25 & 4.13 & 4.46 & 0.70 & 0.52 & 19.16 & 2.16 & 11.75 & 0.90 & 18.65 \\
\hline EPIC 203856244 & 6.08 & 5.94 & 6.31 & 0.44 & 0.34 & 4.07 & 1.95 & 12.11 & 0.50 & 23.49 \\
\hline EPIC 203850605 & 4.85 & 4.85 & 4.85 & 0.60 & 0.60 & 3.92 & 3.52 & 10.76 & 0.00 & 16.86 \\
\hline EPIC 203115615 & 4.73 & 4.58 & 5.02 & 0.65 & 0.45 & 26.17 & 0.99 & 12.36 & 1.00 & 28.58 \\
\hline EPIC 202615424 & 4.30 & 4.30 & 4.30 & 0.52 & 0.52 & 2.82 & 3.69 & 12.34 & 0.00 & 18.47 \\
\hline EPIC 203873374 & 5.23 & 5.20 & 5.26 & 0.48 & 0.46 & 2.06 & 0.63 & 12.50 & 0.10 & 26.99 \\
\hline
\end{tabular}

Notes. The table lists: colour-corrected integrated $(V-K)_{0}$ colour, colours, masses, and rotation periods for the primary and secondary components, respectively; I magnitude of the whole system, and magnitude difference between the two components. ${ }^{(a)}$ Values taken from Rebull et al. (2018). ${ }^{(b)}$ Values taken from Tokovinin \& Briceño (2018). 